\title{
A POSTERIORI ESTIMATES OF INVERSE OPERATORS FOR BOUNDARY VALUE PROBLEMS IN LINEAR ELLIPTIC PARTIAL DIFFERENTIAL EQUATIONS
}

\author{
YOSHITAKA WATANABE, TAKEHIKO KINOSHITA, AND MITSUHIRO T. NAKAO
}

\begin{abstract}
This paper presents constructive a posteriori estimates of inverse operators for boundary value problems in linear elliptic partial differential equations (PDEs) on a bounded domain. This type of estimate plays an important role in the numerical verification of the solutions for boundary value problems in nonlinear elliptic PDEs. In general, it is not easy to obtain the a priori estimates of the operator norm for inverse elliptic operators. Even if we can obtain these estimates, they are often over estimated. Our proposed a posteriori estimates are based on finite-dimensional spectral norm estimates for the Galerkin approximation and expected to converge to the exact operator norm of inverse elliptic operators. This provides more accurate estimates, and more efficient verification results for the solutions of nonlinear problems.
\end{abstract}

\section{INTRODUCTION}

The main aim of this paper is to provide the positive constant $C_{L^{2}, H_{0}^{1}}$ satisfying the operator norm:

$$
\left\|(-\Delta+b \cdot \nabla+c)^{-1}\right\|_{\mathcal{L}\left(L^{2}(\Omega), H_{0}^{1}(\Omega)\right)} \leq C_{L^{2}, H_{0}^{1}}
$$

Here, $\Omega \subset \mathbb{R}^{d}(d=1,2,3)$ is a bounded polygonal or polyhedral domain, $b \in$ $L^{\infty}(\Omega)^{d}, c \in L^{\infty}(\Omega) . \quad H_{0}^{1}(\Omega):=\left\{u \in H^{1}(\Omega) ; u=0\right.$ on $\left.\partial \Omega\right\}$ is a Hilbert space with respect to the inner product $(u, v)_{H_{0}^{1}(\Omega)}:=(\nabla u, \nabla v)_{L^{2}(\Omega)^{d}}$ and the norm is $\|u\|_{H_{0}^{1}(\Omega)}:=(u, u)_{H_{0}^{1}(\Omega)}^{\frac{1}{2}}$. The constant $C_{L^{2}, H_{0}^{1}}$ plays an essential role in the verification of the solutions for the boundary value problems in nonlinear elliptic partial differential equations (PDEs) [8, 9] and must be numerically determined.

By defining $\mathcal{L}:=-\Delta+b \cdot \nabla+c$, the problem of obtaining the estimates of (1.1) is equivalent to the norm estimation of the solution $u$ for the following boundary value problems in linear elliptic PDEs such that

$$
\begin{cases}\mathcal{L} u=f, & \text { in } \Omega, \\ u=0, & \text { on } \partial \Omega,\end{cases}
$$

Received by the editor May 18, 2011.

2010 Mathematics Subject Classification. Primary 65N30, 35J25; Secondary 65N15, 35 B45.

Key words and phrases. Constructive a posteriori estimates, Galerkin method, linear elliptic PDEs.

This work was supported by a Grant-in-Aid from the Ministry of Education, Culture, Sports, Science and Technology of Japan (No. 20224001, No. 21540134) and supported by Kyoto University Mathematics Global COE Program. 
for arbitrary $f \in L^{2}(\Omega)$. Here, the weak solution $u \in H_{0}^{1}(\Omega)$ of (1.2a) and (1.2b) is defined by the following variational equation:

$$
L(u, v)=(f, v)_{L^{2}(\Omega)}, \quad \forall v \in H_{0}^{1}(\Omega),
$$

for a bilinear form $L: H_{0}^{1}(\Omega) \times H_{0}^{1}(\Omega) \rightarrow \mathbb{R}$ defined by

$$
L(u, v):=(\nabla u, \nabla v)_{L^{2}(\Omega)^{d}}+((b \cdot \nabla) u, v)_{L^{2}(\Omega)}+(c u, v)_{L^{2}(\Omega)} .
$$

If we assume the coercivity of $L$, then by the Lax-Milgram theorem, there exists a unique solution for (1.3), indicating the existence of the inverse of $\mathcal{L}$. Nakao, Hashimoto, Watanabe 6] proposed the validated computational technique that demonstrates the existence of $\mathcal{L}^{-1}$ even if the coercivity of $L$ is not assumed. They also derived a technique for obtaining the estimates of (1.1). In Section 3 , we introduce these results and discuss them in more detail.

However, the estimates of $\mathcal{L}^{-1}$ in [6] have an unavoidable lower bound. In this study, we propose a novel technique to obtain a posteriori estimates of (1.1) using $\mathcal{L}_{h}^{-1}$ which is defined by the Galerkin approximate integral operator for $\mathcal{L}^{-1}$. Our new approach has no restricted lower bound; therefore, it is expected that we can obtain $C_{L^{2}, H_{0}^{1}}$ smaller than that of [6]. Moreover, we introduce a posteriori error estimates for $\mathcal{L}^{-1}$ and $\mathcal{L}_{h}^{-1}$.

The contents of this paper are as follows: In Section 2, we introduce the necessary function spaces and calculate the a priori error estimates for their Galerkin approximations. In Section 3, we present previously reported methods of error estimation. In Section 4, we propose a posteriori estimates of (1.1). In Section 5, we propose a posteriori error estimates for $\mathcal{L}^{-1}$ and $\mathcal{L}_{h}^{-1}$. Note that in this study, the term "a posteriori error estimates" is defined as the operator norm for integral operators. This suggests that these error estimates can be calculated whenever the Galerkin approximate spaces are given. Therefore, they do not depend on $f$. In Section [6, we compare the constants given by [6] and propose a new value of $C_{L^{2}, H_{0}^{1}}$ for the test problems.

\section{FunCtion SPACES AND GALERKIN APPROXIMATION}

In this section, we introduce the function spaces and constructive error estimates of projections to finite-dimensional subspaces. Let $X(\Omega):=\left\{u \in L^{2}(\Omega)\right.$; $\left.\Delta u \in L^{2}(\Omega)\right\}$ be a Banach space with respect to the norm $\|u\|_{X(\Omega)}:=$ $\|u\|_{L^{2}(\Omega)}+\|\Delta u\|_{L^{2}(\Omega)}$. We again define the linear elliptic partial differential operator $\mathcal{L}: H_{0}^{1}(\Omega) \cap X(\Omega) \rightarrow L^{2}(\Omega)$ by $\mathcal{L}:=-\Delta+b \cdot \nabla+c$. The norms of Banach space $L^{\infty}(\Omega)^{d}$ and $L^{\infty}(\Omega)$ are defined by

$$
\|b\|_{L^{\infty}(\Omega)^{d}}:=\operatorname{esssup}_{x \in \Omega} \sqrt{b_{1}(x)^{2}+\cdots+b_{d}(x)^{2}}, \quad\|c\|_{L^{\infty}(\Omega)}:=\operatorname{ess}_{x \in \Omega}|c(x)| .
$$

The following theorem is the Sobolev inequality.

Theorem 2.1 (Sobolev inequality). Let the constant $p$ satisfy $1 \leq p \leq 2^{*}$, where $2^{*}$ is the Sobolev conjugate index defined by $2^{*}:=\frac{2 d}{d-2}$. Then, there exists a positive constant $C_{s, p}>0$ such that

$$
\|u\|_{L^{p}(\Omega)} \leq C_{s, p}\|u\|_{H_{0}^{1}(\Omega)}, \quad \forall u \in H_{0}^{1}(\Omega) .
$$


Let $S_{h}(\Omega)$ be an approximate finite-dimensional subspace of $H_{0}^{1}(\Omega)$ dependent on the parameter $h$. For example, $S_{h}(\Omega)$ is considered to be a finite element subspace with the mesh size $h$ or a set of the finite polynomial expansion with polynomial degree. Let $n$ be a degree of freedom for $S_{h}(\Omega)$ and $\phi_{i}$ the basis function of $S_{h}(\Omega)$. This indicates that $S_{h}(\Omega):=\operatorname{span}_{1 \leq i \leq n}\left\{\phi_{i}\right\}$.

We denote the symmetric positive definite matrices $D_{\phi}$ and $L_{\phi}$ in $\mathbb{R}^{n, n}$ by

$$
\begin{aligned}
D_{\phi, i, j} & :=\left(\nabla \phi_{j}, \nabla \phi_{i}\right)_{L^{2}(\Omega)^{d}}, \quad 1 \leq i, j \leq n, \\
L_{\phi, i, j} & :=\left(\phi_{j}, \phi_{i}\right)_{L^{2}(\Omega)}, \quad 1 \leq i, j \leq n .
\end{aligned}
$$

Let $D_{\phi}^{1 / 2}$ and $L_{\phi}^{1 / 2}$ be the Cholesky factors of $D_{\phi}$ and $L_{\phi}$, respectively, i.e.,

$$
D_{\phi}=D_{\phi}^{1 / 2} D_{\phi}^{T / 2} \text { and } \quad L_{\phi}=L_{\phi}^{1 / 2} L_{\phi}^{T / 2} .
$$

We define the $H_{0}^{1}$ projection $P_{h}^{1}: H_{0}^{1}(\Omega) \rightarrow S_{h}(\Omega)$ by

$$
\left(u-P_{h}^{1} u, v_{h}\right)_{H_{0}^{1}(\Omega)}=0, \quad \forall v_{h} \in S_{h}(\Omega) .
$$

Therefore, the problems of the solvability of the variational equation (2.4) and the nonsingularity of $D_{\phi}$ become equivalent. Because the matrix $D_{\phi}$ is positive definite, the projection $P_{h}^{1}$ is well defined. Similarly, we define the $L^{2}$ projection $P_{h}^{0}: L^{2}(\Omega) \rightarrow S_{h}(\Omega)$ by

$$
\left(u-P_{h}^{0} u, v_{h}\right)_{L^{2}(\Omega)}=0, \quad \forall v_{h} \in S_{h}(\Omega) .
$$

Now, we assume that the following estimates of $P_{h}^{1}$ hold.

Assumption 2.2. There exist a positive constant $C(h)>0$ satisfying

$$
\begin{gathered}
\left\|u-P_{h}^{1} u\right\|_{H_{0}^{1}(\Omega)} \leq C(h)\|\Delta u\|_{L^{2}(\Omega)}, \quad \forall u \in H_{0}^{1}(\Omega) \cap X(\Omega), \\
\left\|u-P_{h}^{1} u\right\|_{L^{2}(\Omega)} \leq C(h)\left\|u-P_{h}^{1} u\right\|_{H_{0}^{1}(\Omega)}, \quad \forall u \in H_{0}^{1}(\Omega) .
\end{gathered}
$$

Assumption 2.2 is the most basic of error estimates in the Galerkin method. For example, in the case of a finite element space using piecewise bilinear polynomial approximation of $H_{0}^{1}(\Omega)$, the value $C(h)$ is known by $C(h)=\frac{h}{\pi}$. Alternatively, in the case of piecewise biquadratic polynomial approximation, Assumption 2.2 is satisfied by $C(h)=\frac{h}{2 \pi}$. Moreover, these approximations give the optimal constants (e.g., [5]). If $N$ degree polynomial approximation is used, Assumption 2.2 is satisfied by $C(h)=O\left(\frac{h}{N}\right)$. However, in these cases, the optimal constants are unknown (e.g., [3]).

For arbitrary $f \in L^{2}(\Omega)$, we define the Galerkin approximate solution $u_{h} \in$ $S_{h}(\Omega)$ of (1.3) such that

$$
\begin{aligned}
\left(\nabla u_{h}, \nabla v_{h}\right)_{L^{2}(\Omega)^{d}}+\left((b \cdot \nabla) u_{h}, v_{h}\right)_{L^{2}(\Omega)}+\left(c u_{h}, v_{h}\right)_{L^{2}(\Omega)} & =\left(f, v_{h}\right)_{L^{2}(\Omega)}, \\
& \forall v_{h} \in S_{h}(\Omega) .
\end{aligned}
$$

Let $G_{\phi}$ be a matrix in $\mathbb{R}^{n, n}$, where each element is defined by

$G_{\phi, i, j}:=L\left(\phi_{j}, \phi_{i}\right)=\left(\nabla \phi_{j}, \nabla \phi_{i}\right)_{L^{2}}+\left((b \cdot \nabla) \phi_{j}, \phi_{i}\right)_{L^{2}}+\left(c \phi_{j}, \phi_{i}\right)_{L^{2}}, \quad 1 \leq i, j \leq n$.

Then, the nonsingularity of $G_{\phi}$ and the unique existence of the solution $u_{h}$ in (2.8) become equivalent. Therefore, we assume the nonsingularity of $G_{\phi}$. However, 
when applying the proposed a posteriori estimates, it is necessary to confirm the nonsingularity of $G_{\phi}$ by validated computations.

Next, we define the $L$ projection $P_{h}^{L}: H_{0}^{1}(\Omega) \rightarrow S_{h}(\Omega)$ by

$$
L\left(u-P_{h}^{L} u, v_{h}\right)=0, \quad \forall v_{h} \in S_{h}(\Omega) .
$$

From the nonsingularity of $G_{\phi}, P_{h}^{L}$ is well defined. If for an arbitrary $f \in L^{2}(\Omega)$ there exists $u$ that is a unique solution for (1.3), then we denote the operator $\mathcal{L}^{-1}$ : $L^{2}(\Omega) \rightarrow H_{0}^{1}(\Omega)$ by $u=\mathcal{L}^{-1} f$. By defining the operator $\mathcal{L}_{h}^{-1}: L^{2}(\Omega) \rightarrow S_{h}(\Omega)$, we obtain $u_{h}$, the solution of (2.8). Thus, we obtain $\mathcal{L}_{h}^{-1}=P_{h}^{L} \mathcal{L}^{-1}$ from the definition of $P_{h}^{L}$.

\section{KNOWN RESULTS}

In this section, we introduce the result for the invertibility condition of the operator $\mathcal{L}$ and its previously determined estimates. We define the following constants:

$$
\begin{aligned}
C_{1} & :=\|b\|_{L^{\infty}(\Omega)^{d}}+C_{s, 2}\|c\|_{L^{\infty}(\Omega)}, \\
K_{1}(h) & :=C(h)\left(C_{s, 2}\|\operatorname{div} b\|_{L^{\infty}(\Omega)}+C_{1}\right), \\
C_{2} & :=\|b\|_{L^{\infty}(\Omega)^{d}}+C(h)\|c\|_{L^{\infty}(\Omega)}, \\
K_{2}(h) & :=\sqrt{d} C_{s, 2}\|b\|_{L^{\infty}(\Omega)^{d}}+C(h) C_{s, 2}\|c\|_{L^{\infty}(\Omega)}, \\
M_{\phi}^{11}(h) & :=\left\|D_{\phi}^{T / 2} G_{\phi}^{-1} D_{\phi}^{1 / 2}\right\|_{2},
\end{aligned}
$$

where $\|\cdot\|_{2}$ is the matrix two-norm, i.e., the maximum singular value.

Theorem 3.1 ([6, Theorem 2.1 \& Corollary 1]). Let $K(h)>0$ be defined by

$$
K(h):= \begin{cases}K_{1}(h), & \text { if } b \in W^{1, \infty}(\Omega)^{d}, \\ K_{2}(h), & \text { if } b \in L^{\infty}(\Omega)^{d}\end{cases}
$$

Let $\kappa_{\phi}>0$ satisfy

$$
\kappa_{\phi}:=C(h)\left(C_{1} M_{\phi}^{11}(h) K(h)+C_{2}\right)<1 .
$$

Then, under Assumption 2.2 , the operator $\mathcal{L}$ is invertible.

We define the symmetric positive definite matrix $R$ in $\mathbb{R}^{2,2}$ by

$$
R:=\frac{1}{\left(1-\kappa_{\phi}\right)^{2}}\left(\begin{array}{cc}
M_{\phi}^{11}(h)^{2}\left(C_{1}^{2} C(h)^{2}+\left(1-C_{2} C(h)\right)^{2}\right) & \text { symmetry } \\
M_{\phi}^{11}(h)\left(C_{1} C(h)+\left(1-C_{2} C(h)\right) M_{\phi}^{11}(h) K(h)\right) & 1+M_{\phi}^{11}(h)^{2} K(h)^{2}
\end{array}\right) .
$$

We can obtain the estimates of $\mathcal{L}^{-1}$ using $R$.

Theorem 3.2 ([6, Theorem 2.3]). By using the same assumptions as those in Theorem 3.1, we obtain the following estimates,

$$
\left\|\mathcal{L}^{-1}\right\|_{\mathcal{L}\left(L^{2}(\Omega), H_{0}^{1}(\Omega)\right)} \leq C_{s, 2}\|R\|_{2}^{\frac{1}{2}}
$$

Even if $b$ has sufficient regularity, the estimate (3.3) is expected to converge to $C_{s, 2} \max \left\{M_{\phi}^{11}, 1\right\}$ as $h \rightarrow 0$. As a result, this a posteriori method overestimates the operator norm and fails to converge to its exact operator norm. Further discussion of the error in the previously reported a posteriori estimates for $\mathcal{L}^{-1}$ and $\mathcal{L}_{h}^{-1}$ are discussed in 7]. Next, we will improve this estimation method (3.3), and propose the new a posteriori estimates of $\mathcal{L}^{-1}$ which converge to the exact operator norm. 
Theorem 3.3 ([7, Theorem 6]). By using the same assumptions as those in Theorem 3.1, we obtain the following error estimates:

$$
\left\|\mathcal{L}^{-1}-\mathcal{L}_{h}^{-1}\right\|_{\mathcal{L}\left(L^{2}(\Omega), H_{0}^{1}(\Omega)\right.} \leq C(h) \frac{1+C_{s, 2} M_{\phi}^{11}(h) C_{1}}{1-\kappa_{\phi}} \sqrt{1+\left(M_{\phi}^{11}(h) K(h)\right)^{2}},
$$

$$
\left\|\mathcal{L}^{-1}-\mathcal{L}_{h}^{-1}\right\|_{\mathcal{L}\left(L^{2}(\Omega), L^{2}(\Omega)\right)} \leq C(h) \frac{1+C_{s, 2} M_{\phi}^{11}(h) C_{1}}{1-\kappa_{\phi}}\left(C(h)+C_{s, 2} M_{\phi}^{11}(h) K(h)\right) .
$$

The proof of Theorem 3.3 can be obtained by using the proof of Theorem 3.2 . Therefore, if the estimates of (3.3) can be improved, then the error estimates of Theorem 3.3 can also be improved. In Section 6, we use numerical examples to describe the results of improving these error estimates.

Remark 3.4 (Aubin-Nitsche trick). In the case of $b \in W^{1, \infty}(\Omega)^{d}$, the convergence order of (3.5) is $O\left(h^{2}\right)$ because we can apply the $L^{2}$ error estimates by applying the Aubin-Nitsche trick, the convergence order of $K(h)\left(=K_{1}(h)\right)$ is $O(h)$. On the other hand, in the case of $b \in L^{\infty}(\Omega)^{d}$ and $b \notin W^{1, \infty}(\Omega)^{d}$, the convergence order of (3.5) is $O(h)$. Because the solution for the dual problem of (1.2a) and (1.2b) does not have sufficient regularity, we cannot apply the Aubin-Nitsche trick. Therefore, $K(h)\left(=K_{2}(h)\right)$ does not have the order of $h$. Thus, when the dual problem becomes singular, it is difficult to obtain the $L^{2}$ error estimates whose convergence order is $O\left(h^{2}\right)$. To address this difficulty, we have previously proposed a technique for obtaining $L^{2}$ error estimates by using validated computations in [4]. When this technique is used, it is expected that $K_{2}(h)$ will have the order $h$.

\section{A POSTERIORI ESTIMATES FOR INVERSE LINEAR ELLIPTIC OPERATORS}

In this section, we improve the previously reported estimates of (3.3) by proposing the new a posteriori estimates of $\mathcal{L}^{-1}$, which converge to the exact operator norm. To this end, let $M_{\phi}^{00}(h), M_{\phi}^{10}(h)$, and $M_{\phi}^{01}(h)$ be the positive constants defined by

$$
\begin{gathered}
M_{\phi}^{00}(h):=\left\|L_{\phi}^{T / 2} G_{\phi}^{-1} L_{\phi}^{1 / 2}\right\|_{2}, \quad M_{\phi}^{10}(h):=\left\|D_{\phi}^{T / 2} G_{\phi}^{-1} L_{\phi}^{1 / 2}\right\|_{2}, \\
M_{\phi}^{01}(h):=\left\|L_{\phi}^{T / 2} G_{\phi}^{-1} D_{\phi}^{1 / 2}\right\|_{2},
\end{gathered}
$$

respectively. The following lemma consists of the constants $M_{\phi}^{00}$ and $M_{\phi}^{10}$.

Lemma 4.1. The operator norm of $\mathcal{L}_{h}^{-1}$ satisfies the following equalities:

$$
\begin{aligned}
\left\|\mathcal{L}_{h}^{-1}\right\|_{\mathcal{L}\left(L^{2}(\Omega), L^{2}(\Omega)\right)} & =M_{\phi}^{00}(h), \\
\left\|\mathcal{L}_{h}^{-1}\right\|_{\mathcal{L}\left(L^{2}(\Omega), H_{0}^{1}(\Omega)\right)} & =M_{\phi}^{10}(h) .
\end{aligned}
$$

Proof. Note that we only discuss the proof of (4.2). The proof of (4.1) is omitted because it is almost the same. For arbitrary $f \in L^{2}(\Omega)$, let $u_{h}:=\mathcal{L}_{h}^{-1} f \in S_{h}(\Omega)$. The values from $u_{h}$ to $P_{h}^{0} f$ are the elements of $S_{h}(\Omega)$, and can be expressed by the linear combination of the basis of $S_{h}(\Omega)$. This indicates that $\alpha:=\left(\alpha_{1}, \cdots, \alpha_{n}\right)^{T}$ 
and $\beta:=\left(\beta_{1}, \cdots, \beta_{n}\right)^{T} \in \mathbb{R}^{n}$ exists such that

$$
u_{h}(x)=\sum_{i=1}^{n} \alpha_{i} \phi_{i}(x), \quad P_{h}^{0} f(x)=\sum_{i=1}^{n} \beta_{i} \phi_{i}(x) .
$$

The equation (2.8) is rewritten using $\alpha$ and $\beta$ to give

$$
G_{\phi} \alpha=L_{\phi} \beta,
$$

where the matrices $G_{\phi}$ and $L_{\phi}$ are defined by (2.9) and (2.3), respectively. Because $L_{\phi}$ and $D_{\phi}$ are symmetric positive definite matrices, they can be factorized by the Cholesky decomposition. From (4.3), we have

$$
\begin{aligned}
\left\|u_{h}\right\|_{H_{0}^{1}(\Omega)}^{2} & =\alpha^{T} D_{\phi} \alpha=\left(D_{\phi}^{T / 2} \alpha\right)^{T}\left(D_{\phi}^{T / 2} \alpha\right) \\
\left\|u_{h}\right\|_{H_{0}^{1}(\Omega)} & =\left\|D_{\phi}^{T / 2} \alpha\right\|_{2} \\
& =\left\|\left(D_{\phi}^{T / 2} G_{\phi}^{-1} L_{\phi}^{1 / 2}\right)\left(L_{\phi}^{T / 2} \beta\right)\right\|_{2} \\
& \leq\left\|D_{\phi}^{T / 2} G_{\phi}^{-1} L_{\phi}^{1 / 2}\right\|_{2}\left\|L_{\phi}^{T / 2} \beta\right\|_{2} \\
& =\left\|D_{\phi}^{T / 2} G_{\phi}^{-1} L_{\phi}^{1 / 2}\right\|_{2}\left\|P_{h}^{0} f\right\|_{L^{2}(\Omega)} \\
& \leq\left\|D_{\phi}^{T / 2} G_{\phi}^{-1} L_{\phi}^{1 / 2}\right\|_{2}\|f\|_{L^{2}(\Omega)} .
\end{aligned}
$$

Therefore, we obtain

$$
\left\|\mathcal{L}_{h}^{-1}\right\|_{\mathcal{L}\left(L^{2}(\Omega), H_{0}^{1}(\Omega)\right)}=\sup _{L^{2}(\Omega) \ni f \neq 0} \frac{\left\|\mathcal{L}_{h}^{-1} f\right\|_{H_{0}^{1}(\Omega)}}{\|f\|_{L^{2}(\Omega)}} \leq\left\|D_{\phi}^{T / 2} G_{\phi}^{-1} L_{\phi}^{1 / 2}\right\|_{2} .
$$

Next, we consider the existence of $f_{0} \in L^{2}(\Omega)$ which satisfies the equalities (4.4) and (4.5). Let $B_{\phi}:=D_{\phi}^{T / 2} G_{\phi}^{-1} L_{\phi}^{1 / 2}$, let $\lambda>0$ be a maximum eigenvalue of $B_{\phi}^{T} B_{\phi}$, and $\gamma \neq 0$ an eigenvector associated to $\lambda$. Note that $\lambda$ satisfies $\sqrt{\lambda}=$ $\left\|D_{\phi}^{T / 2} G_{\phi}^{-1} L_{\phi}^{1 / 2}\right\|_{2}$. Because $L_{\phi}^{T / 2}$ is nonsingular, we denote $\beta_{0}:=\left(L_{\phi}^{T / 2}\right)^{-1} \gamma$. Let $f_{0} \in S_{h}(\Omega)$ be defined by $f_{0}:=\sum_{i=1}^{n} \beta_{0, i} \phi_{i}$. Then, $f_{0}$ satisfies the equalities (4.4) and (4.5). Therefore, we obtain the equality of (4.4) by

$$
\begin{aligned}
\left\|B_{\phi} L_{\phi}^{T / 2} \beta_{0}\right\|_{2}^{2} & =\gamma^{T} B_{\phi}^{T} B_{\phi} \gamma \\
& =\lambda\|\gamma\|_{2}^{2} \\
\left\|B_{\phi} L_{\phi}^{T / 2} \beta_{0}\right\|_{2} & =\left\|D_{\phi}^{T / 2} G_{\phi}^{-1} L_{\phi}^{1 / 2}\right\|_{2}\|\gamma\|_{2} .
\end{aligned}
$$

Furthermore, $P_{h}^{0} f_{0}=f_{0}$ is clear from $f_{0} \in S_{h}(\Omega)$. Therefore, we have the equality of (4.5). As a result, (4.6) satisfies the equality.

From Lemma 4.1, we can expect that accurate estimates of $\mathcal{L}^{-1}$ can be obtained using $M_{\phi}^{10}(h)$. Therefore, we have the following theorem.

Theorem 4.2. Let $\hat{\kappa}_{\phi}>0$ satisfy

$$
\hat{\kappa}_{\phi}:=C(h) C_{2}\left(1+M_{\phi}^{10}(h) C_{1}\right)<1 .
$$


Then under the same assumptions as those in Theorem 3.1, we have the following estimates:

$$
\left\|\mathcal{L}^{-1}\right\|_{\mathcal{L}\left(L^{2}(\Omega), H_{0}^{1}(\Omega)\right.} \leq \frac{\sqrt{M_{\phi}^{10}(h)^{2}+C(h)^{2}\left(1+M_{\phi}^{10}(h) C_{1}\right)^{2}}}{1-\hat{\kappa}_{\phi}} .
$$

Proof. By assuming (3.2), we find that the bounded linear operator $\mathcal{L}^{-1}: L^{2}(\Omega) \rightarrow$ $H_{0}^{1}(\Omega) \cap X(\Omega)$ exists. For arbitrary $f \in L^{2}(\Omega)$, let $u:=\mathcal{L}^{-1} f \in H_{0}^{1}(\Omega) \cap X(\Omega)$. By using the definition of $u, u$ satisfies the following integral equation,

$$
u=(-\Delta)^{-1}(-(b \cdot \nabla) u-c u+f),
$$

where $(-\Delta)^{-1}: L^{2}(\Omega) \rightarrow H_{0}^{1}(\Omega) \cap X(\Omega)$ denotes the solution operator of the Poisson equation with homogeneous Dirichlet boundary conditions. We can decompose the finite- and infinite-dimensional parts using the projection $P_{h}^{1}$ such that

$$
\left\{\begin{array}{l}
P_{h}^{1} u=P_{h}^{1}(-\Delta)^{-1}(-(b \cdot \nabla) u-c u+f), \\
\left(I-P_{h}^{1}\right) u=\left(I-P_{h}^{1}\right)(-\Delta)^{-1}(-(b \cdot \nabla) u-c u+f) .
\end{array}\right.
$$

In short, we denote $u_{\perp}:=u-P_{h}^{1} u$. From (4.9a), for arbitrary $v_{h} \in S_{h}(\Omega)$, we obtain

$$
\begin{aligned}
\left(\nabla P_{h}^{1} u, \nabla v_{h}\right)_{L^{2}(\Omega)^{d}} & =\left(\nabla P_{h}^{1}(-\Delta)^{-1}(-(b \cdot \nabla) u-c u+f), \nabla v_{h}\right)_{L^{2}(\Omega)^{d}} \\
& =\left(-(b \cdot \nabla) u-c u+f, v_{h}\right)_{L^{2}(\Omega)}, \quad \text { therefore } \\
L\left(P_{h}^{1} u, v_{h}\right) & =\left(-(b \cdot \nabla) u_{\perp}-c u_{\perp}+f, v_{h}\right)_{L^{2}(\Omega)} \\
& =\left(P_{h}^{0}\left(-(b \cdot \nabla) u_{\perp}-c u_{\perp}+f\right), v_{h}\right)_{L^{2}(\Omega)} .
\end{aligned}
$$

Because $P_{h}^{1} u$ and $P_{h}^{0}\left(-(b \cdot \nabla) u_{\perp}-c u_{\perp}+f\right)$ are the elements of $S_{h}(\Omega)$, they are expressible by the linear combination of the basis of $S_{h}(\Omega)$. This indicates that $\alpha:=\left(\alpha_{1}, \cdots, \alpha_{n}\right)^{T}$ and $\beta:=\left(\beta_{1}, \cdots, \beta_{n}\right)^{T} \in \mathbb{R}^{n}$ exist such that

$$
P_{h}^{1} u=\sum_{i=1}^{n} \alpha_{i} \phi_{i}, \quad P_{h}^{0}\left(-(b \cdot \nabla) u_{\perp}-c u_{\perp}+f\right)=\sum_{i=1}^{n} \beta_{i} \phi_{i} .
$$

(4.10) is rewritten using $\alpha$ and $\beta$ to give

$$
G_{\phi} \alpha=L_{\phi} \beta \text {. }
$$

From (4.11), we have

$$
\begin{aligned}
\left\|P_{h}^{1} u\right\|_{H_{0}^{1}(\Omega)}^{2} & =\alpha^{T} D_{\phi} \alpha \\
& =\left(D_{\phi}^{T / 2} \alpha\right)^{T}\left(D_{\phi}^{T / 2} G_{\phi}^{-1} L_{\phi}^{1 / 2}\right)\left(L_{\phi}^{T / 2} \beta\right) \\
& \leq\left\|P_{h}^{1} u\right\|_{H_{0}^{1}(\Omega)}\left\|D_{\phi}^{T / 2} G_{\phi}^{-1} L_{\phi}^{1 / 2}\right\|_{2}\left\|P_{h}^{0}\left(-(b \cdot \nabla) u_{\perp}-c u_{\perp}+f\right)\right\|_{L^{2}(\Omega)} .
\end{aligned}
$$

By using Assumption 2.2 and the fact that $P_{h}^{0}$ is an $L^{2}$ projection, we have

$$
\begin{aligned}
\left\|P_{h}^{1} u\right\|_{H_{0}^{1}(\Omega)} & \leq M_{\phi}^{10}(h)\left\|-(b \cdot \nabla) u_{\perp}-c u_{\perp}+f\right\|_{L^{2}(\Omega)} \\
& \leq M_{\phi}^{10}(h)\left(\|b\|_{L^{\infty}(\Omega)^{d}}\left\|\nabla u_{\perp}\right\|_{L^{2}(\Omega)^{d}}+\|c\|_{L^{\infty}(\Omega)}\left\|u_{\perp}\right\|_{L^{2}(\Omega)}+\|f\|_{L^{2}(\Omega)}\right) \\
& \leq M_{\phi}^{10}(h) C_{2}\left\|\nabla u_{\perp}\right\|_{L^{2}(\Omega)^{d}}+M_{\phi}^{10}(h)\|f\|_{L^{2}(\Omega)} .
\end{aligned}
$$


Next, by calculating the $H_{0}^{1}$ norm of (4.9b) from Assumption 2.2, we obtain

$$
\begin{aligned}
\left\|u_{\perp}\right\|_{H_{0}^{1}(\Omega)} \leq C(h)\|-(b \cdot \nabla) u-c u+f\|_{L^{2}(\Omega)} & \\
\leq & C(h)\left(\|b\|_{L^{\infty}(\Omega)^{d}}\|\nabla u\|_{L^{2}(\Omega)^{d}}+\|c\|_{L^{\infty}(\Omega)}\|u\|_{L^{2}(\Omega)}+\|f\|_{L^{2}(\Omega)}\right) \\
\leq & \quad+\| c(h)\left(\|b\|_{L^{\infty}}\left(\left\|\nabla P_{h}^{1} u\right\|_{L^{2}}+\left\|\nabla u_{\perp}\right\|_{L^{2}}\right)\right. \\
& \left.\quad+\|c\|_{L^{\infty}}\left(\left\|P_{h}^{1} u\right\|_{L^{2}}+\left\|u_{\perp}\right\|_{L^{2}}\right)+\|f\|_{L^{2}}\right) \\
\leq & C(h) C_{1}\left\|\nabla P_{h}^{1} u\right\|_{L^{2}(\Omega)^{d}}+C(h) C_{2}\left\|\nabla u_{\perp}\right\|_{L^{2}(\Omega)^{d}}+C(h)\|f\|_{L^{2}(\Omega)} .
\end{aligned}
$$

From (4.13) and (4.12), we obtain

$$
\begin{aligned}
\left\|u_{\perp}\right\|_{H_{0}^{1}} \leq & C(h) C_{1}\left(M_{\phi}^{10}(h) C_{2}\left\|u_{\perp}\right\|_{H_{0}^{1}}+M_{\phi}^{10}(h)\|f\|_{L^{2}}\right) \\
& +C(h) C_{2}\left\|u_{\perp}\right\|_{H_{0}^{1}}+C(h)\|f\|_{L^{2}} .
\end{aligned}
$$

By using assumption (4.7), we obtain

$$
\left\|u_{\perp}\right\|_{H_{0}^{1}(\Omega)} \leq C(h) \frac{1+M_{\phi}^{10}(h) C_{1}}{1-\hat{\kappa}_{\phi}}\|f\|_{L^{2}(\Omega)} .
$$

From (4.12) and (4.14), we have

$$
\left\|P_{h}^{1} u\right\|_{H_{0}^{1}(\Omega)} \leq \frac{M_{\phi}^{10}(h)}{1-\hat{\kappa}_{\phi}}\|f\|_{L^{2}(\Omega)} .
$$

Finally, from (4.15), (4.14), and the fact that $P_{h}^{1}$ is an $H_{0}^{1}$ projection, we have

$$
\begin{aligned}
\|u\|_{H_{0}^{1}(\Omega)}^{2} & =\left\|P_{h}^{1} u\right\|_{H_{0}^{1}(\Omega)}^{2}+\left\|u_{\perp}\right\|_{H_{0}^{1}(\Omega)}^{2} \\
& \leq \frac{M_{\phi}^{10}(h)^{2}}{\left(1-\hat{\kappa}_{\phi}\right)^{2}}\|f\|_{L^{2}(\Omega)}^{2}+C(h)^{2} \frac{\left(1+M_{\phi}^{10}(h) C_{1}\right)^{2}}{\left(1-\hat{\kappa}_{\phi}\right)^{2}}\|f\|_{L^{2}(\Omega)}^{2} \\
& =\frac{M_{\phi}^{10}(h)^{2}+C(h)^{2}\left(1+M_{\phi}^{10}(h) C_{1}\right)^{2}}{\left(1-\hat{\kappa}_{\phi}\right)^{2}}\|f\|_{L^{2}(\Omega)}^{2} .
\end{aligned}
$$

Therefore, this proof is completed. 4.2 .

The $L^{2}$ estimates are obtained by providing a proof similar to that of Theorem

Theorem 4.3. By using the same assumptions as those in Theorem 4.2, we obtain the following estimates:

$$
\left\|\mathcal{L}^{-1}\right\|_{\mathcal{L}\left(L^{2}(\Omega), L^{2}(\Omega)\right)} \leq \frac{M_{\phi}^{00}(h)+C(h)^{2}\left(1+M_{\phi}^{10}(h) C_{1}\right)}{1-\hat{\kappa}_{\phi}} .
$$

Proof. For arbitrary $f \in L^{2}(\Omega)$, let $u:=\mathcal{L}^{-1} f \in H_{0}^{1}(\Omega) \cap X(\Omega)$. From (4.11), we obtain

$$
\begin{aligned}
\left\|P_{h}^{1} u\right\|_{L^{2}(\Omega)}^{2} & =\left(L_{\phi}^{T / 2} \alpha\right)^{T}\left(L_{\phi}^{T / 2} G_{\phi}^{-1} L_{\phi}^{1 / 2}\right)\left(L_{\phi}^{T / 2} \beta\right) \\
& \leq\left\|P_{h}^{1} u\right\|_{L^{2}(\Omega)}\left\|L_{\phi}^{T / 2} G_{\phi}^{-1} L_{\phi}^{1 / 2}\right\|_{2}\left\|P_{h}^{0}\left(-(b \cdot \nabla) u_{\perp}-c u_{\perp}+f\right)\right\|_{L^{2}(\Omega)} .
\end{aligned}
$$


By using Assumption 2.2 and (4.14), we obtain

$$
\begin{aligned}
\left\|P_{h}^{1} u\right\|_{L^{2}(\Omega)} & \leq M_{\phi}^{00}(h) C_{2}\left\|\nabla u_{\perp}\right\|_{L^{2}(\Omega)^{d}}+M_{\phi}^{00}(h)\|f\|_{L^{2}(\Omega)} \\
& \leq M_{\phi}^{00}(h) C_{2} C(h) \frac{1+M_{\phi}^{10}(h) C_{1}}{1-\hat{\kappa}_{\phi}}\|f\|_{L^{2}(\Omega)}+M_{\phi}^{00}(h)\|f\|_{L^{2}(\Omega)} \\
& =\frac{M_{\phi}^{00}(h)}{1-\hat{\kappa}_{\phi}}\|f\|_{L^{2}(\Omega)} .
\end{aligned}
$$

Similarly, for the estimates of $\left\|u_{\perp}\right\|_{L^{2}(\Omega)}$, by using Assumption 2.2 and (4.14), we obtain

$$
\left\|u_{\perp}\right\|_{L^{2}(\Omega)} \leq C(h)\left\|u_{\perp}\right\|_{H_{0}^{1}(\Omega)} \leq C(h)^{2} \frac{1+M_{\phi}^{10}(h) C_{1}}{1-\hat{\kappa}_{\phi}}\|f\|_{L^{2}(\Omega)} .
$$

From (4.17) and (4.18), we obtain

$$
\begin{aligned}
\|u\|_{L^{2}(\Omega)} & \leq\left\|P_{h}^{1} u\right\|_{L^{2}(\Omega)}+\left\|u_{\perp}\right\|_{L^{2}(\Omega)} \\
& \leq \frac{M_{\phi}^{00}(h)}{1-\hat{\kappa}_{\phi}}\|f\|_{L^{2}(\Omega)}+C(h)^{2} \frac{1+M_{\phi}^{10}(h) C_{1}}{1-\hat{\kappa}_{\phi}}\|f\|_{L^{2}(\Omega)} .
\end{aligned}
$$

Therefore, this proof is completed.

To obtain the $L^{p}$ estimates, the following theorem is necessary.

Theorem 4.4 (Gagliardo-Nirenberg). Let the constants $p$ and $q$ satisfy $1 \leq p \leq$ $q^{*} \leq \infty$. Then, for arbitrary $0 \leq \theta \leq 1$, there exists the positive constant $C_{g, r, p, q}>$ 0 such that

$$
\|u\|_{L^{r}(\Omega)} \leq C_{g, r, p, q}\|u\|_{L^{p}(\Omega)}^{\theta}\|u\|_{W^{1, q}(\Omega)}^{1-\theta}, \quad \forall u \in W^{1, q}(\Omega),
$$

where $\frac{1}{r}=\frac{\theta}{p}+\frac{1-\theta}{q^{*}}$.

It is known that the optimal constants of $C_{g, r, p, q}$ in Theorem 4.4 become the minimum eigenvalue of certain nonlinear elliptic boundary value problems (e.g., [1]). Moreover, we can obtain the upper bounds of $C_{g, r, p, q}$ by Sobolev constants. For example, if we can calculate the Sobolev constants for $C_{s, 2^{*}}>0$ in (2.1), then for arbitrary $2 \leq p \leq 2^{*}$, we obtain

$$
\begin{aligned}
\|u\|_{L^{p}(\Omega)} & \leq\|u\|_{L^{2}(\Omega)}^{1-d\left(\frac{1}{2}-\frac{1}{p}\right)}\|u\|_{L^{2^{*}}(\Omega)}^{d\left(\frac{1}{2}-\frac{1}{p}\right)} \\
& \leq C_{s, 2^{*}}^{d\left(\frac{1}{2}-\frac{1}{p}\right)}\|u\|_{L^{2}(\Omega)}^{1-d\left(\frac{1}{2}-\frac{1}{p}\right)}\|u\|_{H_{0}^{1}(\Omega)}^{d\left(\frac{1}{2}-\frac{1}{p}\right)} .
\end{aligned}
$$

Therefore, we obtain $C_{g, p, 2,2} \leq C_{s, 2^{*}}^{d\left(\frac{1}{2}-\frac{1}{p}\right)}$.

Finally, in this section, we present the $L^{p}$ estimates.

Corollary 4.5. Assume that the following two inequalities are provided:

$$
\begin{aligned}
& \left\|\mathcal{L}^{-1}\right\|_{\mathcal{L}\left(L^{2}(\Omega), L^{2}(\Omega)\right)} \leq C_{L^{2}, L^{2}}, \\
& \left\|\mathcal{L}^{-1}\right\|_{\mathcal{L}\left(L^{2}(\Omega), H_{0}^{1}(\Omega)\right)} \leq C_{L^{2}, H_{0}^{1}},
\end{aligned}
$$

then, for arbitrary $2 \leq p \leq 2^{*}$, we obtain

$$
\left\|\mathcal{L}^{-1}\right\|_{\mathcal{L}\left(L^{2}(\Omega), L^{p}(\Omega)\right)} \leq C_{g, p, 2,2} C_{L^{2}, L^{2}}^{1-d\left(\frac{1}{2}-\frac{1}{p}\right)} C_{L^{2}, H_{0}^{1}}^{d\left(\frac{1}{p}-\frac{1}{1}\right)}
$$


Proof. For arbitrary $f \in L^{2}(\Omega)$, let $u:=\mathcal{L}^{-1} f \in H_{0}^{1}(\Omega) \cap X(\Omega)$. From GagliardoNirenberg inequality and assumptions, we have

$$
\begin{aligned}
\|u\|_{L^{p}(\Omega)} & \leq C_{g, p, 2,2}\|u\|_{L^{2}(\Omega)}^{1-d\left(\frac{1}{2}-\frac{1}{p}\right)}\|u\|_{H_{0}^{1}(\Omega)}^{d\left(\frac{1}{2}-\frac{1}{p}\right)} \\
& \leq C_{g, p, 2,2} C_{L^{2}, L^{2}}^{1-d\left(\frac{1}{2}-\frac{1}{p}\right)}\|f\|_{L^{2}(\Omega)}^{1-d\left(\frac{1}{2}-\frac{1}{p}\right)} C_{L^{2}, H_{0}^{1}}^{d\left(\frac{1}{2}-\frac{1}{p}\right)}\|f\|_{L^{2}(\Omega)}^{d\left(\frac{1}{2}-\frac{1}{p}\right)} .
\end{aligned}
$$

Therefore, this proof is completed.

\section{A POSTERIORI ERROR ESTIMATES FOR INVERSE LINEAR ELLIPTIC OPERATORS}

In this section, we consider the error estimates for $\mathcal{L}^{-1}$ and $\mathcal{L}_{h}^{-1}$. We obtain the following estimates corresponding to $P_{h}^{1}$ and $P_{h}^{L}$.

Lemma 5.1. We obtain the following error estimates:

$$
\begin{gathered}
\left\|P_{h}^{1} u-P_{h}^{L} u\right\|_{L^{2}(\Omega)} \leq M_{\phi}^{01}(h)\left\|P_{h}^{1} \Delta^{-1}(b \cdot \nabla+c)\left(u-P_{h}^{1} u\right)\right\|_{H_{0}^{1}(\Omega)}, \\
\forall u \in H_{0}^{1}(\Omega), \\
\left\|P_{h}^{1} u-P_{h}^{L} u\right\|_{H_{0}^{1}(\Omega)} \leq M_{\phi}^{11}(h)\left\|P_{h}^{1} \Delta^{-1}(b \cdot \nabla+c)\left(u-P_{h}^{1} u\right)\right\|_{H_{0}^{1}(\Omega)}, \\
\forall u \in H_{0}^{1}(\Omega) .
\end{gathered}
$$

Proof. For arbitrary $u \in H_{0}^{1}(\Omega)$, let $u_{\perp}:=u-P_{h}^{1} u$ and $g:=\Delta^{-1}(b \cdot \nabla+c) u_{\perp}$. For arbitrary $v_{h} \in S_{h}(\Omega)$, we have

$$
\begin{aligned}
L\left(P_{h}^{1} u-P_{h}^{L} u, v_{h}\right) & =-\left((b \cdot \nabla+c) u_{\perp}, v_{h}\right)_{L^{2}(\Omega)} \\
& =\left(\nabla \Delta^{-1}(b \cdot \nabla+c) u_{\perp}, \nabla v_{h}\right)_{L^{2}(\Omega)^{d}} \\
& =\left(\nabla P_{h}^{1} g, \nabla v_{h}\right)_{L^{2}(\Omega)^{d}} .
\end{aligned}
$$

Because $P_{h}^{1} u-P_{h}^{L} u$ and $P_{h}^{1} g$ are the elements of $S_{h}(\Omega)$, they can be expressed by the linear combination of the basis of $S_{h}(\Omega)$. This indicates that $\alpha:=\left(\alpha_{1}, \cdots, \alpha_{n}\right)^{T}$ and $\beta:=\left(\beta_{1}, \cdots, \beta_{n}\right)^{T} \in \mathbb{R}^{n}$ exists such that

$$
P_{h}^{1} u-P_{h}^{L} u=\sum_{i=1}^{n} \alpha_{i} \phi_{i}, \quad P_{h}^{1} g=\sum_{i=1}^{n} \beta_{i} \phi_{i} .
$$

Equation (5.3) is written using $\alpha$ and $\beta$ to give

$$
G_{\phi} \alpha=D_{\phi} \beta \text {. }
$$

Therefore, we have the following $L^{2}$ error estimates

$$
\begin{aligned}
\left\|P_{h}^{1} u-P_{h}^{L} u\right\|_{L^{2}(\Omega)}^{2} & =\alpha^{T} L_{\phi} \alpha \\
& =\left(L_{\phi}^{T / 2} \alpha\right)^{T}\left(L_{\phi}^{T / 2} G_{\phi}^{-1} D_{\phi}^{1 / 2}\right)\left(D_{\phi}^{T / 2} \beta\right) \\
& \leq\left\|P_{h}^{1} u-P_{h}^{L} u\right\|_{L^{2}(\Omega)}\left\|L_{\phi}^{T / 2} G_{\phi}^{-1} D_{\phi}^{1 / 2}\right\|_{2}\left\|P_{h}^{1} g\right\|_{H_{0}^{1}(\Omega)} .
\end{aligned}
$$


Similarly, we have the following $H_{0}^{1}$ error estimates

$$
\begin{aligned}
\left\|P_{h}^{1} u-P_{h}^{L} u\right\|_{H_{0}^{1}(\Omega)}^{2} & =\alpha^{T} D_{\phi} \alpha \\
& =\left(D_{\phi}^{T / 2} \alpha\right)^{T}\left(D_{\phi}^{T / 2} G_{\phi}^{-1} D_{\phi}^{1 / 2}\right)\left(D_{\phi}^{T / 2} \beta\right) \\
& \leq\left\|P_{h}^{1} u-P_{h}^{L} u\right\|_{H_{0}^{1}(\Omega)}\left\|D_{\phi}^{T / 2} G_{\phi}^{-1} D_{\phi}^{1 / 2}\right\|_{2}\left\|P_{h}^{1} g\right\|_{H_{0}^{1}(\Omega)} .
\end{aligned}
$$

Therefore, this proof is completed.

Theorem 5.2. By using the same assumptions as those in Theorem 4.2, we obtain the following error estimates:

$$
\begin{aligned}
\left\|\mathcal{L}^{-1}-\mathcal{L}_{h}^{-1}\right\|_{\mathcal{L}\left(L^{2}(\Omega), H_{0}^{1}(\Omega)\right.} \leq C(h) \frac{1+M_{\phi}^{10}(h) C_{1}}{1-\hat{\kappa}_{\phi}} \sqrt{1+\left(M_{\phi}^{11}(h) K(h)\right)^{2}} \\
\left\|\mathcal{L}^{-1}-\mathcal{L}_{h}^{-1}\right\|_{\mathcal{L}\left(L^{2}(\Omega), L^{2}(\Omega)\right)} \leq C(h) \frac{1+M_{\phi}^{10}(h) C_{1}}{1-\hat{\kappa}_{\phi}}\left(C(h)+M_{\phi}^{01}(h) K(h)\right) .
\end{aligned}
$$

Proof. For arbitrary $f \in L^{2}(\Omega)$, let $u:=\mathcal{L}^{-1} f \in H_{0}^{1}(\Omega) \cap X(\Omega)$ and $u_{h}:=\mathcal{L}_{h}^{-1} f \in$ $S_{h}(\Omega)$. By the definition of $u$ and $u_{h}$, we have $u_{h}=P_{h}^{L} u$. Let $u_{\perp}:=u-P_{h}^{1} u$.

First, we derive (5.4). By using the definition of an $H_{0}^{1}$ projection and (5.2), we have

$$
\begin{aligned}
\left\|u-u_{h}\right\|_{H_{0}^{1}(\Omega)}^{2} & =\left\|u-P_{h}^{1} u\right\|_{H_{0}^{1}(\Omega)}^{2}+\left\|P_{h}^{1} u-P_{h}^{L} u\right\|_{H_{0}^{1}(\Omega)}^{2} \\
& \leq\left\|u-P_{h}^{1} u\right\|_{H_{0}^{1}(\Omega)}^{2}+M_{\phi}^{11}(h)^{2}\left\|P_{h}^{1} \Delta^{-1}(b \cdot \nabla+c) u_{\perp}\right\|_{H_{0}^{1}(\Omega)}^{2} .
\end{aligned}
$$

Then, from [4, Theorem 3.3.], we obtain the following estimates:

$$
\left\|P_{h}^{1} \Delta^{-1}(b \cdot \nabla+c)\left(u-P_{h}^{1} u\right)\right\|_{H_{0}^{1}(\Omega)} \leq K(h)\left\|u-P_{h}^{1} u\right\|_{H_{0}^{1}(\Omega)} .
$$

Furthermore, from (4.14), we obtain

$$
\left\|u-u_{h}\right\|_{H_{0}^{1}(\Omega)} \leq \sqrt{1+\left(M_{\phi}^{11}(h) K(h)\right)^{2}} C(h) \frac{1+M_{\phi}^{10}(h) C_{1}}{1-\hat{\kappa}_{\phi}}\|f\|_{L^{2}(\Omega)} .
$$

Therefore, we obtain (5.4).

Next, we derive (5.5). By using Assumption 2.2 and (5.1), we obtain

$$
\begin{aligned}
& \left\|u-u_{h}\right\|_{L^{2}(\Omega)} \leq\left\|u-P_{h}^{1} u\right\|_{L^{2}(\Omega)}+\left\|P_{h}^{1} u-P_{h}^{L} u\right\|_{L^{2}(\Omega)} \\
& \quad \leq C(h)\left\|u-P_{h}^{1} u\right\|_{H_{0}^{1}(\Omega)}+M_{\phi}^{01}(h)\left\|P_{h}^{1} \Delta^{-1}(b \cdot \nabla+c)\left(u-P_{h} u\right)\right\|_{H_{0}^{1}(\Omega)} .
\end{aligned}
$$

From (5.6) and (4.14), we have

$$
\begin{aligned}
\left\|u-u_{h}\right\|_{L^{2}(\Omega)} & \leq\left(C(h)+M_{\phi}^{01}(h) K(h)\right)\left\|u-P_{h}^{1} u\right\|_{H_{0}^{1}(\Omega)} \\
& \leq\left(C(h)+M_{\phi}^{01}(h) K(h)\right) C(h) \frac{1+M_{\phi}^{10}(h) C_{1}}{1-\hat{\kappa}_{\phi}}\|f\|_{L^{2}(\Omega)} .
\end{aligned}
$$

Therefore, we obtain (5.5).

Finally, in this section, we present the $L^{p}$ error estimates. 
Corollary 5.3. Assume that the following two inequalities are provided:

$$
\begin{aligned}
\left\|\mathcal{L}^{-1}-\mathcal{L}_{h}^{-1}\right\|_{\mathcal{L}\left(L^{2}(\Omega), L^{2}(\Omega)\right.} & \leq E_{L^{2}, L^{2}}, \\
\left\|\mathcal{L}^{-1}-\mathcal{L}_{h}^{-1}\right\|_{\mathcal{L}\left(L^{2}(\Omega), H_{0}^{1}(\Omega)\right)} & \leq E_{L^{2}, H_{0}^{1}} .
\end{aligned}
$$

For arbitrary $2 \leq p \leq 2^{*}$, we have

$$
\left\|\mathcal{L}^{-1}-\mathcal{L}_{h}^{-1}\right\|_{\mathcal{L}\left(L^{2}(\Omega), L^{p}(\Omega)\right)} \leq C_{g, p, 2,2} E_{L^{2}, L^{2}}^{1-d\left(\frac{1}{2}-\frac{1}{p}\right)} E_{L^{2}, H_{0}^{1}}^{d\left(\frac{1}{p}-\frac{1}{1}\right)} .
$$

The proof is similar to Corollary 4.5 .

Remark 5.4. From the results of Lemma 4.1 and Theorem $5.2, M_{\phi}^{00}(h)$ and $M_{\phi}^{10}(h)$ converge to $\left\|\mathcal{L}^{-1}\right\|_{\mathcal{L}\left(L^{2}(\Omega), L^{2}(\Omega)\right)}$ and $\left\|\mathcal{L}^{-1}\right\|_{\mathcal{L}\left(L^{2}(\Omega), H_{0}^{1}(\Omega)\right)}$ as $h \rightarrow 0$, respectively.

\section{NUMERICAL EXAMPLE}

In this section, we apply the described method to numerical experiments on test problems. First, we compared (4.8) with (3.3). For simplicity, in this section, the domain $\Omega$ is fixed as the unit square $(0,1) \times(0,1) \subset \mathbb{R}^{2}$. We assume that the finite element partition of $\Omega$ is a uniform triangular mesh and the basis of $S_{h}(\Omega)$ is a set of piecewise linear polynomials (P1 element). Therefore, Assumption 2.2 is realized by $C(h)=0.49293 h$ (e.g., [2]).

6.1. Steady-state convection diffusion equation. We show a computational result in the case of the steady-state convection diffusion equation $\mathcal{L}=-\Delta+b \cdot \nabla+c$. In particular, we consider $b\left(x_{1}, x_{2}\right)=5\left(-x_{2}+\frac{1}{2}, x_{1}-\frac{1}{2}\right)^{T} \in W^{1, \infty}(\Omega)^{2}$.

TABle 1. Convection diffusion equation for $c=0$.

\begin{tabular}{ccccccc}
\hline $1 / h$ & $M_{\phi}^{00}(h)$ & $M_{\phi}^{01}(h)$ & $M_{\phi}^{10}(h)$ & $M_{\phi}^{11}(h)$ & $(3.3)$ & $(4.8)$ \\
\hline 10 & 0.04943 & 0.22232 & 0.22232 & 1.00002 & 2.12100 & 0.34720 \\
20 & 0.05034 & 0.22435 & 0.22435 & 1.00001 & 1.73512 & 0.27102 \\
30 & 0.05051 & 0.22473 & 0.22473 & 1.00001 & 1.63742 & 0.25304 \\
40 & 0.05057 & 0.22487 & 0.22487 & 1.00001 & 1.59292 & 0.24512 \\
50 & 0.05060 & 0.22493 & 0.22493 & 1.00001 & 1.56748 & 0.24068 \\
\hline
\end{tabular}

First, we consider the case of $c=0$. Table 1 shows its verification results. The column for $1 / h$ lists the reciprocal number of the mesh size. These values denote the number of partitions for the domain $\Omega$. From $b \neq 0$, the matrix $G_{\phi}$ is nonsymmetric and has complex eigenvalues. However, $M_{\phi}^{01}(h)$ and $M_{\phi}^{10}(h)$ are always equal. Our proposed new estimates in (4.8) are smaller than previous estimates (3.3) for any mesh size.

Next, we consider the case of $c=-10$; Table 2 shows its verification results. In this table, "fail" denotes that the invertibility condition failed in Theorem 3.1 The same tendency as Table 1 is seen for this problem. 
TABLE 2. Convection diffusion equation for $c=-10$.

\begin{tabular}{ccccccc}
\hline $1 / h$ & $M_{\phi}^{00}(h)$ & $M_{\phi}^{01}(h)$ & $M_{\phi}^{10}(h)$ & $M_{\phi}^{11}(h)$ & $(3.3)$ & $(4.8)$ \\
\hline 10 & 0.09772 & 0.43953 & 0.43953 & 1.97692 & fail & fail \\
20 & 0.10133 & 0.45167 & 0.45167 & 2.01302 & 6.81287 & 0.95000 \\
30 & 0.10203 & 0.45400 & 0.45400 & 2.01996 & 5.09800 & 0.69895 \\
40 & 0.10228 & 0.45482 & 0.45482 & 2.02241 & 4.53489 & 0.61676 \\
50 & 0.10239 & 0.45520 & 0.45520 & 2.02355 & 4.25499 & 0.57604 \\
\hline
\end{tabular}

6.2. Linearized semilinear equation. We show a computational result in the case of linearized equation of the following semilinear PDEs:

$$
\begin{cases}-\Delta u=\lambda\left(1+u+u^{2}-a u^{3}\right) & \text { in } \Omega, \\ u=0, & \text { on } \partial \Omega,\end{cases}
$$

where $\lambda>0$ and $0 \leq a \leq 1$ are constants. For the constant parameters $\lambda$ and $a$, it is known that (6.1a) and (6.1b) have at least two positive solutions; we denote them as the upper and lower solutions, respectively. Let $u_{h}$ be the finite element solutions for (6.1a) and (6.1b). This indicates that $u_{h} \in S_{h}(\Omega)$ satisfies the following variational equation:

$$
\left(\nabla u_{h}, \nabla v_{h}\right)_{L^{2}(\Omega)^{2}}=\lambda\left(1+u_{h}+u_{h}^{2}-a u_{h}^{3}, v_{h}\right)_{L^{2}(\Omega)}, \quad \forall v_{h} \in S_{h}(\Omega) .
$$

The finite element solutions $u_{h}$ were obtained by the Newton-Raphson method using usual floating point arithmetic. Then, the linearized operator at $u_{h}$ is defined by $\mathcal{L}=-\Delta+\lambda\left(1+2 u_{h}-3 a u_{h}^{2}\right)$. We introduce the operator norm estimates for the inverse linearized operator $\mathcal{L}^{-1}$.

TABLE 3. Linearized semilinear equation at the upper approximate solution.

\begin{tabular}{ccccccc}
\hline $1 / h$ & $M_{\phi}^{00}(h)$ & $M_{\phi}^{01}(h)$ & $M_{\phi}^{10}(h)$ & $M_{\phi}^{11}(h)$ & $(3.3)$ & $\underline{4.8}$ \\
\hline 10 & 0.07082 & 0.32622 & 0.32622 & 2.19839 & 10.34011 & 0.83100 \\
20 & 0.07297 & 0.33356 & 0.33356 & 2.22458 & 2.89776 & 0.40142 \\
30 & 0.07338 & 0.33498 & 0.33498 & 2.22970 & 2.50199 & 0.36286 \\
40 & 0.07353 & 0.33547 & 0.33547 & 2.23150 & 2.38044 & 0.35076 \\
50 & 0.07360 & 0.33571 & 0.33571 & 2.23234 & 2.32652 & 0.34538 \\
\hline
\end{tabular}

Table 3 shows the verification results of the linearized inverse operator at the upper approximate solution $u_{h}$ for $\lambda=4$ and $a=0.001$. The effectiveness of the method and the validity of our new estimates for this problem are shown.

Table 4 shows the verification results of the linearized inverse operator at the lower approximate solution $u_{h}$ for $\lambda=4$ and $a=0.001$.

Remark 6.1 (Computer environment). All computations were carried out on a Intel Xeon E5520 2.27GHz (OS: Red Hat Enterprise Linux Server release 5.5) by using INTLAB version 6.0, a toolbox in MATLAB R2010a developed by Rump [10] for self-validating algorithms. Therefore, all numerical values in these tables are verified data in the sense of strictly rounding error control. 
TABLE 4. Linearized semilinear equation at the lower approximate solution.

\begin{tabular}{ccccccc}
\hline $1 / h$ & $M_{\phi}^{00}(h)$ & $M_{\phi}^{01}(h)$ & $M_{\phi}^{10}(h)$ & $M_{\phi}^{11}(h)$ & $(3.3)$ & (4.8) \\
\hline 10 & 0.07255 & 0.32630 & 0.32630 & 1.46826 & 1.51285 & 0.34420 \\
20 & 0.07489 & 0.33379 & 0.33379 & 1.48834 & 1.49944 & 0.33828 \\
30 & 0.07534 & 0.33523 & 0.33523 & 1.49223 & 1.49716 & 0.33722 \\
40 & 0.07550 & 0.33574 & 0.33574 & 1.49360 & 1.49638 & 0.33686 \\
50 & 0.07558 & 0.33597 & 0.33597 & 1.49424 & 1.49602 & 0.33669 \\
\hline
\end{tabular}

\section{Conclusion}

We propose a method for constructive a posteriori estimates of inverse operators for boundary value problems. It is particularly notable that as in (4.8) and (4.16), our proposed estimates are expected to converge to the exact operator norm according to Theorem 5.2. By comparing the a posteriori estimates (3.3) in [6] and (4.8) for some test problem, we show that this holds. Our proposed new estimates (4.8) are smaller than the previous estimates (3.3) in the test problems, and more closely reflect the true error.

\section{REFERENCES}

1. M. Agueh, Gagliardo-Nirenberg inequalities involving the gradient $L^{2}$-norm, C. R. Math. Acad. Sci., Paris, 346 (2008), 757-762. MR.2427077 (2009f:49049)

2. F. Kikuchi and X. Liu, Estimation of interpolation error constants for the $P_{0}$ and $P_{1}$ triangular finite elements, Computer methods in applied mechanics and engineering, 196 (2007), 37503758. MR2340000 (2008f:65214)

3. S. Kimura and N. Yamamoto, On explicit bounds in the error for the $H_{0}^{1}$-projection into piecewise polynomial spaces, Bulletin of Informatics and Cybernetics, 31 (1999), No. 2, 109115. MR 1737441

4. T. Kinoshita, K. Hashimoto and M.T. Nakao, On the $L^{2}$ a priori error estimates to the finite element solution of elliptic problems with singular adjoint operator, Numerical Functional Analysis and Optimization, 30 (2009), no. 3-4, 289-305. MR2514218 (2010d:65325)

5. M.T. Nakao, N. Yamamoto and S. Kimura, On the Best Constant in the Error Bound for the $H_{0}^{1}$-Projection into Piecewise Polynomial Spaces, Journal of Approximation Theory, 93 (1998), 491-500. MR.1624846 (99d:41035)

6. M.T. Nakao, K. Hashimoto and Y. Watanabe, A numerical method to verify the invertibility of linear elliptic operators with applications to nonlinear problems, Computing, 75 (2005), 1-14. MR2161437 (2006e:65098)

7. M.T. Nakao and K. Hashimoto, Guaranteed error bounds for finite element approximations of noncoercive elliptic problems and their applications, Journal of Computational and Applied Mathematics, 218 (2008), no. 1, 106-115. MR2431603 (2009e:65181)

8. S. Oishi, Numerical verification of existence and inclusion of solutions for nonlinear operator equations, Journal of Computational and Applied Mathematics, 60 (1995), no. 1-2, 171-185. MR.1354654 (96g:65058)

9. M. Plum, Computer-assisted proofs for semilinear elliptic boundary value problems, Japan Journal of Industrial and Applied Mathematics, 26 (2009), no. 2-3, 419-442. MR2589483 (2011c:35161)

10. S.M. Rump, INTLAB-INTerval LABoratory, in Developments in Reliable Computing, Tibor Csendes, ed., pp. 77-104, Kluwer Academic Publishers, Dordrecht, (1999). http://www.ti3.tuharburg.de/rump/intlab/ 
Research Institute for Information Technology, Kyushu University, Fukuoka 8128581, JAPAN

E-mail address: watanabe@cc.kyushu-u.ac.jp

Research Institute for Mathematical Sciences, Kyoto University, Kyoto 606-8502, JAPAn, Supported by GCOE 'Fostering top LEAdERs in mathematics', Kyoto University

E-mail address: kinosita@kurims.kyoto-u.ac.jp

Sasebo National College of Technology, Nagasaki 857-1193, Japan

E-mail address: mtnakao@post.cc.sasebo.ac.jp 